PROCEEDINGS OF THE

AMERICAN MATHEMATICAL SOCIETY

Volume 130, Number 11, Pages 3171-3176

S 0002-9939(02)06452-3

Article electronically published on April 17, 2002

\title{
BOUNDING THE FITTING HEIGHT OF A SOLVABLE GROUP BY THE NUMBER OF ZEROS IN A CHARACTER TABLE
}

\author{
GUOHUA QIAN
}

(Communicated by Stephen D. Smith)

\begin{abstract}
In this paper, we bound the Fitting height of a solvable group by the number of zeros in a character table.
\end{abstract}

\section{INTRODUCTION}

In this paper, all characters are complex characters and $\operatorname{Irr}(G)$ is the set of irreducible characters of a finite group $G$. An old theorem of Burnside asserts that any nonlinear $\chi \in \operatorname{Irr}(G)$ must vanish at some element of $G$. In this paper, we consider the following problem: given the number of zeros in a character table of a finite group $G$, what can be said about the structure of $G$ ? Although there are many results about zeros of irreducible characters (see [1], [2], [5], [7]), our result is that the Fitting height of a finite solvable group can be bounded by the number of zeros in a character table.

For a finite solvable group $G$, we define characteristic subgroups $F_{i}(G)$ by letting $F_{1}(G)=F(G)$, the unique largest nilpotent normal subgroup of $G$, and $F_{i+1}(G) / F_{i}(G)=F\left(G / F_{i}(G)\right)$. The Fitting height of group $G$, denoted by $n l(G)$, is the smallest number $l$ for which $F_{l}(G)=G$. For a subset $T$ of $G$, let $k_{G}(T)$ be the number of $G$-conjugacy classes contained in $T$. For $\chi \in \operatorname{Irr}(G), v_{G}(\chi)$ denotes the set of elements contained in $G-F(G)$ on which $\chi$ vanishes. It is clear that $v_{G}(\chi)$ is a union of some conjugacy classes of $G$. Let $n v_{G}(\chi)=k_{G}\left(v_{G}(\chi)\right)$, i.e. the number of $G$-conjugacy classes outside $F(G)$ on which $\chi$ vanishes, and let $n v(G)=\max \left\{n v_{G}(\chi) \mid \chi \in \operatorname{Irr}(G)\right\}$. Now our result can be stated as follows.

Theorem 1. Let $G$ be a solvable group. Then $n l(G) \leq(2 n v(G)+5) / 3$.

Note that if we let $m(G)$ be the maximal number of $G$-classes on which some $\chi \in \operatorname{Irr}(G)$ vanishes, then we see that $n v(G) \leq m(G)$ and hence we also get the bound $n l(G) \leq(2 m(G)+5) / 3$.

Throughout this paper, we use the following notations:

$c l_{G}(g):=$ the $G$-conjugacy class containing $g \in G$. Also if we say "class", we always mean "conjugacy class".

$\operatorname{Irr}(G \mid N):=\operatorname{Irr}(G)-\operatorname{Irr}(G / N)$ where $N \triangleleft G$. For a character $\sigma, \operatorname{Irr}(\sigma)$ denotes the set of irreducible constituents of $\sigma$.

Received by the editors May 7, 2001 and, in revised form, June 11, 2001.

2000 Mathematics Subject Classification. Primary 20C15.

Key words and phrases. Solvable group, Frobenius group, irreducible character, conjugacy class.

(C)2002 American Mathematical Society 

ber.

$\Phi(G):=\cap\{M \mid M$ is a maximal subgroup of $G\} ; p$ always denotes a prime num-

\section{Proof of Theorem 1}

In this section, we assume that the letter $G$ always denotes a finite solvable group, and we shall freely use the following known results:

(1) Suppose $G=M N$ is a Frobenius group with kernel $N$ and complement $M$. Then $N$ is nilpotent and $(|M|,|N|)=1$. Furthermore, if $M$ is abelian, then $M$ is cyclic; if 2||$M \mid$, then $N$ is abelian.

(2) Let $N \triangleleft G$ and $\chi \in \operatorname{Irr}(G)$. If $e \chi=\lambda^{G}$ for some $\lambda \in \operatorname{Irr}(N)$ and some positive integer $e$, then $\chi$ vanishes on $G-N$.

(3) Let $N<M$ be two normal subgroups of $G$. If $\psi \in \operatorname{Irr}(M)$ vanishes on $M-N$, then by Clifford's theorem, each member of $\operatorname{Irr}\left(\psi^{G}\right)$ also vanishes on $M-N$.

Lemma 1 ([6. Lemma 18.1]). If $G / F(G)$ is abelian, then there exists $\chi \in \operatorname{Irr}(G)$ such that $\chi(1)=|G: F(G)|$.

Lemma 2. Let $M, N$ be two normal subgroups of $G$ with $1<N<M$. Then:

(1) If $k_{G}(M-N)=1$ and $(|M: N|,|N|)=1$, then $M$ is a Frobenius group with kernel $N$ and prime order complement.

(2) If $G=M$ and $k_{G}(G-N)=1$, then $|G: N|=2$ and $G$ is a Frobenius group with abelian kernel $N$ of odd order.

(3) If $N=F(G)$ and $M / N$ is abelian, then there exists $\psi \in \operatorname{Irr}(M \mid N)$ of degree $|M: N|$ vanishing on $M-N$, and every $\chi \in \operatorname{Irr}\left(\psi^{G}\right)(\subseteq \operatorname{Irr}(G \mid N))$ also vanishes on $M-N$.

Proof. (1) Write $M=H N$, where $H \cong M / N$. Since $k_{G}(M-N)=1$, then $M / N$ is a principal factor of $G$, and hence is an elementary abelian group with prime power order $p^{r}$. Also, we see that all elements in $M-N$ have the same order $p$ since $(|M: N|,|N|)=1$. It implies that for any element $h \in H-\{1\}, C_{M}(h)=H$. Therefore, $M$ is a Frobenius group with abelian complement $H$ so that $H$ is a cyclic group of prime order $p$.

(2) Since $k_{G}(G-N)=1, G / N$ is a principal factor of $G$ and hence $|G / N|=p$. Let $x \in G-N$. Observe that the length of class $c_{G}(x)$ is less than or equal to the order of derived group $G^{\prime}$. It is easy to see that $N=G^{\prime},|G: N|=2$ and $C_{G}(x)=\langle x\rangle$ with order 2. So, $G=\langle x\rangle N$ is a Frobenius group with a complement $\langle x\rangle$ of order 2 , and hence the Frobenius kernel $N$ is an abelian group of odd order.

(3) Since $F(G / \Phi(G))=F(G) / \Phi(G)$, we may assume that $\Phi(G)=1$ and hence $F(G)$ is abelian. Since $F(M)=F(G)$, by Lemma 1 , there exists $\psi \in \operatorname{Irr}(M \mid F(G))$ such that $\psi(1)=|M: F(G)|$. It is clear that $\psi=\lambda^{M}$ for some non-principal linear $\lambda \in \operatorname{Irr}(F(G))$ and hence $\psi$ vanishes on $M-F(G)$. Now for any $\chi \in \operatorname{Irr}\left(\psi^{G}\right)$, we see that $\chi \in \operatorname{Irr}(G \mid F(G))$ and that $\chi$ also vanishes on $M-F(G)$ by Clifford's theorem.

Lemma 3 is somewhat trivial, but is useful and is used over and over in the rest of this paper. Note that Lemma 3 is true for any finite group.

Lemma 3. Let $N \triangleleft G$ and $\psi \in \operatorname{Irr}(G / N)$. Write $\bar{G}=G / N$. Then:

(1) $\operatorname{cl}_{\bar{G}}(\bar{g})$ (viewed as a subset of $G$ ) is again a $G$-class if and only if for every $\chi \in \operatorname{Irr}(G \mid N), \chi(g)=0$; similarly, if $A$ is a union of some cosets of $N$, then $k_{G}(A)=1$ if and only if $k_{\bar{G}}(\bar{A})=1$ and each $\chi \in \operatorname{Irr}(G \mid N)$ vanishes on $A$. 
(2) If $A=v_{\bar{G}}(\psi)$ contains $m$ classes of $G / N$, then $n v_{\bar{G}}(\psi) \leq n v_{G}(\psi)$; with equality if and only if $\chi$ vanishes on $A$ for every $\chi \in \operatorname{Irr}(G \mid N)$; and if $n v_{\bar{G}}(\psi)+1=$ $n v_{G}(\psi)$, then there exists a subset $A_{1}$ of $A$ such that $m-1=k_{G}\left(A_{1}\right)=K_{\bar{G}}\left(A_{1}\right)$, and hence $\chi$ vanishes on $A_{1}$ for every $\chi \in \operatorname{Irr}(G \mid N)$.

(3) $n v(\bar{G}) \leq n v(G)$.

Proof. (1) It follows that $\left|C_{G}(g)\right|=\left|C_{G / N}(g N)\right|+\sum_{\chi \in \operatorname{Irr}(G \mid N)}|\chi(g)|^{2}$ and that $c l_{G / N}(g N)=c l_{G}(g)$ if and only if $\left|C_{G / N}(g N)\right|=\left|C_{G}(g)\right|$.

Statement (2) is immediate from statement (1), and (3) follows from (2).

Lemma 4. Suppose $n l(G) \geq 2$. Write $F(G)=F$ and $F_{2}(G)=F_{2}$. Then:

(1) There exists $T_{0} \triangleleft G$ such that $T_{0}<F, n l\left(G / T_{0}\right)=n l(G)$ and $F / T_{0}$ is a principal factor of $G$.

(2) $n v(G) \geq n v(G / F)+1$.

(3) Let $T \leq F_{2}$ be a normal subgroup of $G$ such that $F \leq T \leq F_{2}$ and $F_{2} / T$ is abelian. If $\left|F_{2}: F\right|$ is not prime, then there exists $\chi \in \operatorname{Irr}(G \mid T)$ such that $\chi$ vanishes on at least two $G$-classes contained in $F_{2}-F$. In particular, if $n l(G) \geq 4$ and $F_{2} / T$ is a principal factor, then the same conclusion holds.

Proof. (1) Let $F / \Phi(G)=D_{1} / \Phi(G) \times \cdots \times D_{s} / \Phi(G)$ be a direct product of minimal normal subgroups $D_{j} / \Phi(G)$. Let $T_{j}=\prod_{k \neq j} D_{k}$. It is clear that there exists some $T_{j}=T_{0}$ such that $n l\left(G / T_{0}\right)=n l(G)$ as desired.

Now we prove conclusions (2) and (3). Let $L \triangleleft G$ maximal such that $L / F$ is an abelian group. By Lemma 2(3), there exists $\chi_{0} \in \operatorname{Irr}(G \mid F)$ such that $\chi_{0}$ vanishes on $L-F$.

(2) It is easy to see that if $n l(G)=2$, then $n v(G) \geq 1$. Suppose $n l(G) \geq 3$ and let $\psi \in \operatorname{Irr}(G / F)$ such that $n v_{G / F}(\psi)=m=n v(G / F)$ and let $A=v_{G / F}(\psi)$. If $n v(G)=n v(G / F)=m$, then $k_{G / F}(A)=m=k_{G}(A)$, and hence each $\chi \in \operatorname{Irr}(G \mid F)$ vanishes on $A$ by Lemma 3. Thus $\chi_{0}$, as a member of $\operatorname{Irr}(G \mid F)$, vanishes not only on $L-F$ but also on $A$. Observe that $A \subseteq G-F_{2}$. It forces that $n v_{G}\left(\chi_{0}\right) \geq m+1$, a contradiction.

(3) Let $G$ be a counterexample. Since $\chi_{0}$ vanishes on $L-F$ and $\chi_{0} \in \operatorname{Irr}(G \mid F) \subseteq$ $\operatorname{Irr}(G \mid T)$, then $k_{G}(L-F)=1$ and hence $L / F$ is a $p$-principal factor of $G$. In particular, $F_{2} / F$ is a $p$-group because $Z\left(F_{2} / F\right) \leq L / F$. Let $N=O_{p}(G)$. Then by Lemma $3(1), k_{G / N}(L / N-F / N)=1$. Now Lemma 2(1) implies that $L / N$ is a Frobenius group with cyclic complement $L / F$ of prime order $p$.

Since $\left|F_{2} / F\right|$ is not a prime, we may choose $M / L$ to be a $p$-principal factor of $G$ with $M \leq F_{2}$. By the assumption of $L$, it is easy to see that $M / F$ is an extra-special $p$-group. Then there exists $\psi \in \operatorname{Irr}(M / F)$ such that $\psi$ vanishes on $M-L$. Now let $\chi_{1} \in \operatorname{Irr}\left(\psi^{G}\right)$; then $\chi_{1}$ also vanishes on $M-L$. Since $\operatorname{ker}\left(\chi_{1}\right)=F$ and $F<T$ (note that we assume $F_{2} / T$ is abelian), we have $\chi_{1} \in \operatorname{Irr}(G \mid T)$. But since $G$ is a counterexample, it forces that $M-L$ is just a class of $G$. Now applying Lemma $3(1)$, we see that $\chi_{0}$ (as a member of $\operatorname{Irr}(G \mid L)$ ) vanishes not only on $L-F$ but also on $M-L$, so $\chi_{0}$ vanishes off two classes contained in $F_{2}-F$, a contradiction.

In particular, if $n l(G) \geq 4$ and $F_{2} / T$ is a principal factor of $G$, then $\left|F_{2}: F\right|$ is not prime because the alternative would force $G / F_{2} \leq A u t\left(F_{2} / F\right)$ to be abelian, and so that the same conclusion holds.

Lemma 5. Suppose $n l(G) \geq 3$. Then $n v(G) \geq 2$ and with equality if and only if $G \cong S_{4}$. 
Proof. Write $F(G)=F, F_{2}(G)=F_{2}$. By Lemma $4(2)$, we have $n v(G) \geq 1+$ $n v(G / F) \geq 2$. It is clear that $n v\left(S_{4}\right)=2$. We now prove $G \cong S_{4}$ provided that $n v(G)=2$ and $n l(G) \geq 3$.

By Lemma 4(2), we see that $n l(G)=3$ and $n v(G / F)=1$.

Step 1. If $F$ is a minimal normal subgroup of $G$, then $G \cong S_{4}$.

We claim that $G / F_{2}$ is abelian. Assume this is not the case. Let $N \triangleleft G$ maximal such that $F_{2} \leq N$ and $G / N$ is not abelian, and let $Z>N$ with $Z / N=Z(G / N)$. By [4. Lemma 12.3], there exists $\phi \in \operatorname{Irr}(G / N)$ such that $\phi$ vanishes on $G-Z$. Since $1=n v(G / F) \geq n v_{G / F}(\phi)$, we have $k_{G / F}(G / F-Z / F)=1$. Now applying Lemma 2(2), we have $|G: Z|=2$. But it is impossible because $|G / Z|=\phi(1)^{2} \neq 2$.

Now $G / F_{2}$ is abelian; by Lemma $2(3)$, there exists $\chi_{1} \in \operatorname{Irr}\left(G / F \mid F_{2} / F\right)$ such that $\chi_{1}$ vanishes on $G-F_{2}$. Since $n v(G / F)=1$ and then $k_{G / F}\left(G / F-F_{2} / F\right)=1$, Lemma 2(2) yields that $G / F$ is a Frobenius group with complement of order 2 and abelian kernel $F_{2} / F$ of odd order. If $k_{G}\left(G-F_{2}\right)=1$, then Lemma $2(2)$ yields that $G$ is a Frobenius group with kernel $F_{2}$ and thus $F_{2}$ is nilpotent, a contradiction. Thus $k_{G}\left(G-F_{2}\right)=2$ and then we easily deduce that $\left|C_{G}(g)\right|=4$ for every $g \in G-F_{2}$. It follows that $F$ is an elementary abelian 2-group.

Let $P \in \operatorname{syl}_{2}(G)$ and let $g \in P-F$. Observe that $\left|P / P^{\prime}\right|=\left|C_{P / P^{\prime}}\left(g P^{\prime}\right)\right|=$ $\sum_{\chi \in \operatorname{Irr}\left(P / P^{\prime}\right)}|\chi(g)|^{2} \leq \sum_{\chi \in \operatorname{Irr}(P)}|\chi(g)|^{2}=\left|C_{P}(g)\right| \leq\left|C_{G}(g)\right|=4$. It follows that $\left|P / P^{\prime}\right|=4$, and hence by O. Taussky's Theorem [3, III, Theorem 11.9], $P$ has a cyclic subgroup with index 2 . Since $F$ is elementary abelian, it forces that $|F| \leq 4$. Now observe that $2^{\prime}$-group $F_{2} / F$ acts faithfully on 2-group $F$. It is easy to check that $\left|F_{2} / F\right|=3$ and $|F|=4$, so that $G \cong S_{4}$, as desired.

Step 2. $G \cong S_{4}$.

By Lemma 4(1), we may choose $T \triangleleft G, T<F$ such that $n l(G / T)=n l(G)=3$ and $F / T$ is a principal factor of $G$. By Lemma 4(2) and Lemma 3(3), we have $n v(G / T)=2$, and then $G / T \cong S_{4}$ by induction and step 1 . Now it suffices to show $T=1$. Suppose $T \neq 1$. To see a contradiction, we may assume that $T$ is a minimal normal subgroup of $G$. Write $\bar{G}=G / T$. It is clear that $F(\bar{G})=\bar{F}, F_{2}(\bar{G})=\bar{F}_{2}$. Applying Lemma 2(3), we may choose $\chi_{1}, \chi_{2} \in \operatorname{Irr}(\bar{G})$ such that $\chi_{1}$ vanishes on $G-F_{2}$ and $\chi_{2}$ vanishes on $F_{2}-F$. Since $2=k_{\bar{G}}\left(\bar{G}-\bar{F}_{2}\right)=n v_{\bar{G}}\left(\chi_{1}\right) \leq n v_{G}\left(\chi_{1}\right) \leq 2$, it follows that $k_{\bar{G}}\left(\bar{G}-\bar{F}_{2}\right)=k_{G}\left(G-F_{2}\right)=2$. By Lemma 3 , each member $\chi$ of $\operatorname{Irr}(G \mid T)$ vanishes on two $G$-classes: $G-F_{2}$, and hence $v_{G}(\chi)=G-F_{2}$ because $n v_{G}(\chi) \leq 2$.

Now we conclude that $\left|C_{G}(g)\right|=\left|C_{\bar{G}}(\bar{g})\right|=4$ for $g \in G-F_{2}$. Note that each member $\chi$ of $\operatorname{Irr}(G \mid T)$ does not vanish on $F_{2}-F$; we have $k_{\bar{G}}\left(\bar{F}_{2}-\bar{F}\right)<k_{G}\left(F_{2}-F\right)$ by Lemma 3 . It follows that $2=k_{G}\left(F_{2}-F\right)$ because $2=1+k_{\bar{G}}\left(\bar{F}_{2}-\bar{F}\right) \leq$ $k_{G}\left(F_{2}-F\right) \leq n v_{G}\left(\chi_{2}\right) \leq 2$.

Since $k_{G}\left(F_{2}-F\right)=2$, it is easy to check that for every $x \in F_{2}-F,\left|C_{G}(x)\right|=6$. Observe that $\left|C_{\bar{G}}(\bar{x})\right|=3$. It forces that $T$ is a 2-group. Now let $P \in \operatorname{syl}_{2}(G)$. Arguing as in Step 1, we see that $\left|P / P^{\prime}\right|=4$ and $P$ has an element of order $|P| / 2$. But $P$ has no such element in $P-F$ (note that for every $g \in P-F,\left|C_{G}(g)\right|=4$ ) or in $F$, a contradiction.

Theorem 1. Let $G$ be a solvable group. Then $n l(G) \leq(2 n v(G)+5) / 3$.

Proof. By Lemma 4 and Lemma 5 , we may assume $n l(G) \geq 4$. Let $G$ be a minimal counterexample with $n l(G)=m+2$. By Lemma 3(3) and Lemma 4(1), we may assume that $\Phi(G)=1$ and $F(G)$ is the unique minimal normal subgroup of $G$ and 
hence $\left(\left|F_{2}(G): F(G)\right|,|F(G)|\right)=1$. For the sake of simplicity, write $F_{3}=F_{3}(G)$, $F_{2}=F_{2}(G)$ and $F=F(G)$.

First we claim that $n l(G) \geq 5$. Suppose $n l(G)=4$ with

$$
4=n l(G)>(2 n v(G)+5) / 3 .
$$

By Lemma 4(2) and Lemma 5 , we see that $n v(G)=3, n v(G / F)=2$ and $G / F \cong S_{4}$. Also, there exists $\chi_{0} \in \operatorname{Irr}\left(G / F_{2}\right)$ such that $\chi_{0}$ vanishes on $G-F_{3}$. Since $n v(G)=3$ and $2=k_{G / F}\left(G / F-F_{3} / F\right) \leq k_{G}\left(G-F_{3}\right) \leq 3$, by Lemma 3, there exists $g_{0} \in G-F_{3}$ such that $c l_{G / F}\left(g_{0} F\right)$ is again a class of $G$ and then $\chi\left(g_{0}\right)=0$ for all $\chi \in \operatorname{Irr}(G \mid F)$. Since $F_{2} / F$ is an abelian group of order 4 , by Lemma $2(3)$, there exists $\chi \in \operatorname{Irr}\left(\psi^{G}\right)$ such that $\chi$ vanishes on $F_{2}-F$, where $\psi \in \operatorname{Irr}\left(F_{2}\right)$ with $\psi(1)=4$. Now we see that $\chi$ vanishes on $g_{0}$ and $F_{2}-F$. If $k_{G}\left(F_{2}-F\right)=1$, then Lemma 2(1) implies that $F_{2}$ is a Frobenius group (note that $\left(\left|F_{2} / F\right|,|F|\right)=1$ as assumed in above paragraph), and so its abelian complement $F_{2} / F$ is cyclic, a contradiction. Now $k_{G}\left(F_{2}-F\right)>1$. Since $n v_{G}(\chi) \leq 3$, it follows that $v_{G}(\chi)=c l_{G}\left(g_{0}\right) \cup\left(F_{2}-F\right)$ and that $\chi$ is an extension of $\psi$ (otherwise, it is easy to check that $\chi$ vanishes on $G-F_{3}$ or $\left.F_{3}-F_{2}\right)$. Now $\chi_{0} \chi \in \operatorname{Irr}(G)$ by [4, Corollary 6.17], and $\chi_{0} \chi$ vanishes on $\left\{G-F_{3}\right\} \cup\left\{F_{2}-F\right\}$ and then $n v(G) \geq n v_{G}\left(\chi_{0} \chi\right) \geq 4$, a contradiction.

By Lemma 4(1), we can choose $T_{1} \triangleleft G, F \leq T_{1}<F_{2}$ such that $n l\left(G / T_{1}\right)=m+1$ and $F_{2} / T_{1}$ is a principal factor of $G$. Similarly, let $K, K_{1} \triangleleft G$ such that $K / T_{1}=$ $F\left(G / T_{1}\right), K_{1} / T_{1}=F_{2}\left(G / T_{1}\right)$, and let $T_{2} \triangleleft G$ with $K \leq T_{2}<K_{1}$ such that $K_{1} / T_{2}$ is a principal factor and $n l\left(G / T_{2}\right)=m$. Write $s=n v\left(G / T_{2}\right)$. By induction, we have $m=n l\left(G / T_{2}\right) \leq(2 s+5) / 3$. Then the hypothesis that $G$ is a counterexample implies $(2 n v(G)+5) / 3<n l(G)=m+2 \leq(2 s+11) / 3$, so that $n v(G) \leq s+2$. Now applying Lemma 3(3) and Lemma 4(2), we conclude $s=n v\left(G / T_{2}\right) \leq n v\left(G / F_{2}\right)<$ $n v(G / F)<n v(G) \leq s+2$, which forces that $n v(G)=s+2, n v(G / F)=s+1$ and $n v\left(G / F_{2}\right)=s$. Because $K / T_{1}$ is the Fitting subgroup of $G / T_{1}$, by Lemma 3(3) and Lemma 4(2), we have $s \leq n v(G / K)<n v\left(G / T_{1}\right) \leq n v(G / F)=s+1$, thus $n v\left(G / T_{1}\right)=s+1$.

Choose $\chi_{2} \in \operatorname{Irr}\left(G / T_{2}\right)$ such that $n v_{G / T_{2}}\left(\chi_{2}\right)=n v\left(G / T_{2}\right)=s$ and let $A=$ $v_{G / T_{2}}\left(\chi_{2}\right)$. Clearly, $A$ (viewed as a subset of $G / T_{1}$ ) is the union of $s$ or $s+1$ $G / T_{1}$-classes because $s+1=n v\left(G / T_{1}\right)$.

Case 1. Suppose $k_{G / T_{1}}(A)=s$.

By Lemma 3, for any $\chi \in \operatorname{Irr}\left(G / T_{1} \mid T_{2} / T_{1}\right), \chi$ vanishes on $A$. Since $n l\left(G / T_{1}\right)=$ $m+1 \geq 4$, by Lemma $4(3)$, there exists $\chi_{1} \in \operatorname{Irr}\left(G / T_{1} \mid T_{2} / T_{1}\right)$ such that $\chi_{1}$ vanishes on at least two $G / T_{1}$-classes contained in $K_{1} / T_{1}-K / T_{1}$. Observe that $A \cap K_{1}=\emptyset$. Since $\chi_{1}$ also vanishes on $A$, we see that $n v\left(G / T_{1}\right) \geq n v_{G / T_{1}}\left(\chi_{1}\right) \geq s+2$, a contradiction.

Case 2. Suppose $k_{G / T_{1}}(A)=s+1$.

Then by Lemma $3(2)$, there exists a subset $A_{1}$ of $A$ such that $k_{G / T_{1}}\left(A_{1}\right)=$ $k_{G / T_{2}}\left(A_{1}\right)=s-1$, and hence for every $\chi \in \operatorname{Irr}\left(G / T_{1} \mid T_{2} / T_{1}\right)$, $\chi$ vanishes on $A_{1}$. By Lemma 4(3), there exists $\chi_{1} \in \operatorname{Irr}\left(G / T_{1} \mid T_{2} / T_{1}\right)$ which vanishes on $C$ : two $G / T_{1}$-classes contained in $K_{1} / T_{1}-K / T_{1}$. Note that $C \cap A=\emptyset$.

Now $\chi_{1}$ vanishes on $s+1 G / T_{1}$-classes: $B=A_{1} \cup C ; \chi_{2}$ vanishes on $s+1$ $G / T_{1}$-classes: $A$. Since $n v_{G}\left(\chi_{1}\right) \leq n v(G)=s+2$, by Lemma $3(2)$, there exists a subset $B_{1}$ of $B$ such that $k_{G}\left(B_{1}\right)=s=k_{G / T_{1}}\left(B_{1}\right)$, and for every $\chi \in \operatorname{Irr}\left(G \mid T_{1}\right), \chi$ vanishes on $B_{1}$. Similarly, there exists $A_{2} \subset A$ which contains $s$ classes of $G$, and for every $\chi \in \operatorname{Irr}\left(G \mid T_{1}\right), \chi$ vanishes on $A_{2}$. Thus for every $\chi \in \operatorname{Irr}\left(G \mid T_{1}\right), \chi$ vanishes on $A_{2} \cup B_{1}$, which contains at least $s+1$ classes of $G$. By Lemma 4(3), there exists 
$\chi_{3} \in \operatorname{Irr}\left(G \mid T_{1}\right)$ which vanishes on at least two $G$-classes $D$ contained in $F_{2}-F$. Note that $\left(A_{2} \cup B_{1}\right) \cap\left(F_{2}-F\right)=\emptyset$. Now $\chi_{3}$ vanishes on at least $s+3 G$-classes: $A_{2} \cup B_{1} \cup D$ and then $n v(G) \geq n v_{G}\left(\chi_{3}\right) \geq s+3$, a contradiction. The proof is complete.

\section{ACKNOWLEDGMENT}

The author thanks Professor Fan Yun for his helpful comments and also the referee for many valuable suggestions.

\section{REFERENCES}

[1] D. Chillag, On zeros of characters of finite groups, Proc. Amer. Math. Soc., 127(1999),977983. MR 99f:20012

[2] S.M. Gagola, Characters vanishing all but two conjugacy classes, Pacific J. Math.109(1983),363-385. MR 85e:20009

[3] B. Huppert, "Endliche gruppen I", Springer-Verlag, Berlin, 1967. MR 37:302

[4] I. M. Isaacs, "Character theory of finite groups", Academic Press, New York, 1976. MR 57:417; corrected reprint CMP 94:14

[5] I.M. Isaacs, G. Navarro and T.R. Wolf, Finite group elements where no irreducible character vanishes, J.Algebra, 222(1999),413-423. MR 2000k:20008

[6] O. Manz and T. R. Wolf, "Representations of solvable groups", Cambridge University press, Cambridge, 1993. MR 95c:20013

[7] G. Navarro, Zeros of primitive characters in solvable groups, J.Algebra,221(1999), 614-650. MR 2000m:20012

Department of Mathematics, Wuhan University, Wuhan 430072, People's Republic of China - And - Department of Mathematics, Changshu College, Jiangsu, 215500, People's Republic OF China

E-mail address: ghqian2000@263.net 